\title{
LA EDUCACIÓN AMBIENTAL EN EL NIVEL EDUCATIVO SUPERIOR DE MANIZALES
}

\author{
LUZ ELENA SEPÚLVEDA GALLEGO \\ lesga@une.net.co
}

Manizales, 2011-06-30 (Rev. 2011-10-31)

\section{RESUMEN}

Con el objetivo de determinar el grado de inclusión de la dimensión ambiental en el quehacer educativo de nivel superior en la ciudad de Manizales, se analizaron los aspectos concernientes a investigación, proyección y formación de profesionales, en las cinco principales universidades de la ciudad, mediante un estudio descriptivo con componente cualitativo y cuantitativo. Se encontró apropiación dispar de las normas relacionadas con investigación y educación ambiental y, pese a que el propósito inicial era elaborar una propuesta para el ámbito local que permitiera incluir la dimensión ambiental en el quehacer universitario, los hallazgos llevaron a realizar una propuesta para el nivel nacional centrada en la modificación de los indicadores y características del proceso de acreditación de alta calidad de los programas.

\section{PALABRAS CLAVE}

Dimensión ambiental, educación ambiental, educación superior.

\section{ENVIRONMENTAL EDUCATION AT THE HIHGER EDUCATION LEVEL IN MANIZALES}

\begin{abstract}
With the objective of determining the degree of inclusion of the environmental dimension in the educational processes at the Higher Education level in Manizales, aspects related to research, projection and education of professionals were analyzed by means of a descriptive study with qualitative and quantitative components. An uneven appropriation of norms related with research and environmental education was found and, despite the initial purpose was to elaborate a proposal for the local setting which allowed the inclusion of the environmental dimension in the university daily process, the findings permitted to submit a proposal for the national level centered in the modification of indicators and characteristics for programs' high quality accreditation processes.
\end{abstract}

\section{KEY WORDS}

Environmental dimension, environmental education, Higher Education.

\section{INTRODUCCIÓN}

Sin detrimento de la autonomía propia de las instituciones de educación superior, la Política Nacional de Educación Ambiental sugiere la inclusión de la dimensión ambiental en los currículos de los diferentes programas, especialmente en aquellos relacionados con la formación de docentes. Considera la Política que, además, estas instituciones deben asumir el liderazgo en la actualización y perfeccionamiento de los 
docentes con la finalidad de lograr claridad en los aspectos conceptuales y fundamentales del quehacer educativo-ambiental; también formar otros agentes educativos ambientales del sector gubernamental, no gubernamental, productivo, periodistas, publicistas y comunicadores en general, asimismo fomentar y apoyar programas y proyectos de investigación en educación ambiental que contribuyan a clarificar las estrategias pedagógicas y didácticas requeridas por su concepción e impacto ideal (Minambiente, MEN, 2002).

Por su parte la Política Nacional de Investigación Ambiental define la investigación ambiental como:

Aquella que se ocupa del estudio del entorno físicobiótico, de su relación con la estructura sociocultural, y de las dinámicas que tal relación conlleva. En tal sentido, la investigación y el conocimiento del ambiente son parte integral de la vida cotidiana de las poblaciones, y deben ser la base para la construcción de las relaciones armónicas con sus respectivos entornos. Sus temáticas cubrirán todos los campos del saber humano orientados a la generación de conocimiento, que coadyuve al logro del desarrollo sostenible como objetivo central de la Política Nacional Ambiental. ( Minambiente, 2001, p.6)

Desde esta perspectiva, en la política se considera que la investigación y la formación ambiental están estrechamente relacionadas y son complementarias. En este marco normativo y como parte de la investigación "La educación ambiental en Manizales: antecedentes y perspectivas ${ }^{2}$ ", se realizó el estudio de la inclusión de la dimensión ambiental en el quehacer educativo del nivel superior de la ciudad de Manizales, específicamente en lo relacionado con las cinco universidades de mayor tamaño y tradición. El propósito inicial era elaborar una propuesta para el ámbito local que permitiera incluir la dimensión ambiental en el quehacer universitario, pero los hallazgos permitieron suponer que la alternativa es nacional y que la clave podría encontrarse en los procesos de acreditación de alta calidad de los programas.

\section{MATERIALES Y MÉTODO}

El estudio realizado fue de tipo descriptivo con componente cualitativo y cuantitativo; se basó en análisis documental y entrevistas.

Se estudiaron las cinco universidades de mayor tamaño y tradición de la ciudad, tres de ellas privadas y dos públicas ${ }^{3}$. Se profundizó en el estudio de la Universidad de Caldas porque en ella se forma la mayoría de los profesionales que ejercen como maestros en los niveles educativos básico y medio.

Los tópicos estudiados fueron las misiones definidas por cada institución, sus políticas y proyectos de investigación, proyección y la caracterización general de sus programas académicos. 


\section{RESULTADOS Y DISCUSIÓN}

Las misiones de las universidades de la ciudad muestran el compromiso con la formación integral de los profesionales; dos de las cinco hacen alusión al compromiso de contribuir con el desarrollo sustentable. La convivencia pacífica, la solidaridad y el pluralismo son otros elementos aludidos en las misiones. Sólo una de las universidades contempla en su misión el aporte a la solución de los problemas regionales, nacionales y a procesos de integración regional, por tanto las cuatro universidades restantes no tuvieron en cuenta en la redacción de su misión el artículo 120 de la Ley de educación superior que dice: "la extensión comprende [...] actividades de servicio tendientes a procurar el bienestar general de la comunidad y la satisfacción de las necesidades de la sociedad" (Colombia, Congreso de la República, 1992).

La política de investigación de una de las universidades muestra el compromiso con realizar aportes para el mejoramiento de la calidad de vida en el marco de la integración del saber, el diálogo entre fe y razón, una preocupación ética y una perspectiva teológica. Otra de ellas busca nuevos rumbos para el desarrollo de la ciencia, la tecnología y la cultura que lleven a la creación de soluciones que respondan a las necesidades del medio regional, para ello se propone cuestionar, reflexionar, explorar, descubrir y proyectar sus saberes; para esta institución "la investigación, se desarrolla dentro de un sentido profundo de la ética científica, desde la cual cuestiona permanentemente la reaccionalidad de su quehacer, con el fin de que la ciencia y la tecnología marchen de la mano con el humanismo y la libertad, en procura del bienestar de todos" liderando procesos de cambio social del país desde el contexto de la región y propiciando la permanente interacción entre los mundos del saber y la vida cotidiana. La tercera universidad fomenta la cultura investigativa, el espíritu científico, el desarrollo del conocimiento y la construcción de saberes en el marco de la disciplinariedad, la interdisciplinariedad y la transdisciplinariedad con una concepción pluralista de la investigación, comprometida con la relevancia social de la misma; en tal sentido, promueve procesos de investigación capaces de apoyar soluciones para los problemas que afectan la región y el país. La cuarta universidad propende por el fortalecimiento del espíritu científico y de desarrollo social mediante procesos investigativos entendidos como "todo proceso continuo y sistemático que pretende la producción de un nuevo conocimiento a nivel general o particular, la innovación y la aplicación tecnológica o el desarrollo de un producto". La última institución promueve y realiza investigación con las finalidades, entre otras, de generar ciencia y actitudes científicas que sirvan de motor de cambio de la sociedad y contribuyan a la consolidación de una cultura fundamentada en el desarrollo científico, aportar a la construcción de un país cohesionado socialmente, con equidad y calidad de vida, contribuir a la consolidación de un sistema de educación que sea patrimonio social y cultural, fundamentado en criterios de calidad.

Veamos ahora la caracterización de los procesos investigativos de estas instituciones de educación superior. El $15 \%$ de las líneas de investigación activas tienen una relación explícita con los asuntos ambientales, de éstas el $76 \%$ contemplan el impacto ambiental y el desarrollo sostenible como variables de peso en su quehacer investigativo. Es mayor la frecuencia de investigaciones de corte biológico que de corte social; no obstante, es importante resaltar que al menos el $48 \%$ de las mismas logra combinar los dos aspectos. El $67 \%$ de los procesos investigativos son reconocidos como interdisciplinarios y en el $55 \%$ de los casos se considera la participación estudiantil en el desarrollo de las investigaciones. 
En las políticas de proyección de las universidades de la ciudad se destacan las estrategias referidas a las prácticas profesionales y sociales, la investigación, las asesorías y consultorías, la organización de eventos de difusión y de educación continuada, la realización de convenios interinstitucionales, el desarrollo comunitario y, en un caso, la orientación pastoral.

En varias de las universidades se entiende la extensión como factor de desarrollo humano y social mediante el cual se puede aportar a la transformación de las realidades local, regional, nacional y mundial, en el marco de principios de equidad y solidaridad. Una de las instituciones la asume como "un sistema de planes y programas sustentable y sostenible de desarrollo social y cultural, económico, político y ambiental de las comunidades locales y regionales, mediante la interacción y la integración permanente de la universidad con el sector estatal, sector no estatal, el sector productivo y la comunidad". Para otra de las instituciones de educación superior de la ciudad la proyección es una forma de concebir, orientar y realizar las acciones propias de la institución, las cuales deben estar "[...] inspiradas en una perspectiva de desarrollo humano que hace énfasis en la formación de personas éticas, críticas e innovadoras [...]"; de esta manera "[...] el conocimiento se aprovecha y se utiliza con la intención de construir ambientes de convivencia pacífica tanto a nivel interno como externo de la institución, con ello se busca propiciar el bien común y el mejoramiento de la calidad de vida de las personas, en el marco de un desarrollo regional sustentable que contemple procesos sociales, económicos, culturales y políticos".

Vista así, la proyección parte de los conocimientos aplicables a la realidad, se compromete con la idea e intención de contribuir a la construcción de pensamiento crítico, innovador y creativo, asume como parte de su responsabilidad social, la necesidad de aportar en la formación de personas éticas, considerando que la universidad cumple un papel importante en el desarrollo social del país.

En este mismo sentido, otra de las universidades considera como principios básicos de la proyección: a) la contribución a la comprensión y solución de problemas nacionales; b) el aprendizaje y la interacción de saberes; c) la responsabilidad social; d) la equidad; e) los valores comunitarios de equidad y de participación social; f) la solidaridad; g) la interacción e integración social; h) la integración académica e institucional, i) la pertinencia social y académica; j) la gestión prospectiva y estratégica, h ) la cooperación; y i) la transparencia.

Varias de las universidades de la ciudad cuentan con oficinas de estudios ambientales: Centro de Estudios Ambientales de la Universidad Autónoma de Manizales; Departamento de Medio Ambiente y Desarrollo de la Universidad de Manizales; Observatorio de Conflictos Ambientales, Departamento de Recursos Naturales y Medio Ambiente y Jardín Botánico de la Universidad de Caldas y, por último, el Instituto de Estudios Ambientales la Universidad Nacional sede Manizales. Todos estos centros se configuran como escenarios propicios para generar procesos de intervención en la problemática ambiental de la región.

De las cinco principales universidades en la ciudad de Manizales, sólo tres contemplan lo ambiental como prioritario en sus políticas formativas; en el plan de estudios del $60 \%$ de los programas académicos se incluye alguna asignatura, módulo o núcleo relacionado con la temática ambiental, pero sólo en el $31 \%$ de ellos se contempla lo ambiental como aspecto relevante y significativo en el quehacer profesional de los futuros egresados. 
El $19 \%$ de las asignaturas, módulos o núcleos relacionados directamente con procesos educativo-ambientales fueron implementados antes de la Constitución Política de 1991. La política universitaria fue el motivo que llevó a la creación del $45 \%$ de estas asignaturas y la iniciativa de los docentes generó el $42 \%$ (en varias de las universidades tales asignaturas son electivas); en el restante $13 \%$ no se tiene claridad respecto a los motivos de creación de las mismas.

En el desarrollo del $77 \%$ de las asignaturas relacionadas con la temática ambiental se parte del fundamento conceptual de entender el ambiente como recurso para administrar, en el $74 \%$ como problema para solucionar, en el 58\% como naturaleza para apreciar, para respetar, para preservar, y, en el $49 \%$ como espacio comunitario para participar. Como es evidente, en la mayoría de los casos se tiene un concepto de ambiente que combina los enunciados anteriormente. Estas categorías fueron enunciadas por Lucié Sauvé (Colombia, MEN, 1994) a finales del siglo pasado y aún siguen siendo vigentes en los procesos de aproximación conceptual a la temática educativo-ambiental.

Las asignaturas responsables de la enseñanza de los asuntos ambientales son teóricas en el $26 \%$ de los casos, teórico-prácticas en el $74 \%$ restante; en éstas las actividades que le dan el componente práctico son salidas de campo (62\%), talleres y procesos investigativos ( $12 \%$ cada uno) laboratorios y trabajo comunitario ( $7 \%$ cada uno).

Las universidades se han preocupado por tener un personal docente calificado, que se configura como un inmenso potencial humano que trabaja en pro de definir colectivamente y de manera disciplinar cuáles son los mecanismos más viables para lograr un proceso de formación permanente en el área ambiental; entre ellos se encuentran postgraduados en agroecología, educación ambiental, derecho ambiental, saneamiento ambiental, sociología ambiental, economía ambiental, gestión y educación ambiental como biotecnología; además se cuenta con docentes e investigadores que, sin especializaciones en el área específica, se encuentran desarrollando diferentes actividades ambientales tales como biólogos, geólogos, ingenieros agrónomos, médicos veterinarios, profesionales en desarrollo familiar, ingenieros civiles, ingenieros forestales, ingenieros químicos, arquitectos, economistas, licenciados en educación ambiental, trabajadores sociales, filósofos, médicos y abogados.

En lo referente a la Universidad de Caldas, su proyecto educativo institucional está permeado por la perspectiva ambiental mediante alusiones explícitas al desarrollo sostenible y sustentable, al equilibrio ambiental, al conocimiento de las problemáticas regionales y nacionales, al estudio y conservación de los bienes naturales y culturales, a la conservación de un ambiente sano, al fomento de la educación y la cultura ecológica, a la educación ambiental en el área de formación general y al desarrollo de nuevos programas académicos directamente relacionados con lo ambiental.

El Estatuto General también involucra aspectos ambientales pues determina que "los objetivos y el desarrollo de los campos de acción de la Universidad, estarán enmarcados en los aspectos sociales, económicos, políticos y culturales de su área de influencia y del país", en tanto puede considerarse que la dimensión ambiental incluye los aspectos sociales, económicos, políticos y culturales mencionados.

El plan de desarrollo 2003-2007 de la institución encontró su justificación en las necesidades de: monitorear la gestión y evaluar la efectividad, la pertinencia y el impacto institucional; enfrentar los riesgos 
derivados de la situación política, social, ecológica y económica del país; identificar los factores internos y externos que influyen sobre el desarrollo de la institución; articular el desarrollo de la universidad con planes, programas y proyectos locales, regionales y nacionales; y generar competencias sociales en términos de una mejor respuesta a la problemática social, económica y ambiental de su área de influencia como del país. Con un evidente compromiso ambiental, en este plan de desarrollo se definieron los valores corporativos de la institución: a) respeto por las distintas formas de vida y la diversidad; b) solidaridad; y c) respeto a la diferencia.

Actualmente, en el plan de desarrollo $2008-2019$ se definió la ambiental como una de sus líneas estratégicas de desarrollo, la cual se apoya en la existencia del Instituto de Investigaciones en Ciencias Ambientales y de la Tierra, que involucra cuatro grupos de investigación. Se reconoce en el documento como debilidad del área, la desarticulación de los trabajos de pequeños grupos de profesores (Universidad de Caldas, 2009, p. 114).

Durante el proceso de preparación para la realización de la primera autoevaluación con fines de acreditación de alta calidad, la Universidad de Caldas incluyó como un nuevo factor de análisis la gestión ambiental, entendida ésta desde cinco parámetros: política ambiental, sistema de gestión ambiental, formación ambiental, gestión de residuos y salud ocupacional.

En el proceso de autoevaluación se determinó la necesidad de intervenir problemas como: a) carencia de cultura y conciencia institucional con respecto a la perspectiva ambiental; b) inadecuado manejo de residuos; c) altos grados de contaminación auditiva y olfativa; d) deficiente conocimiento del quehacer ambiental en la Universidad; e) no aplicación de los propósitos del proyecto educativo institucional referentes a promover la conservación de un ambiente sano y fomentar la educación y cultura ecológica en la universidad; y f) desarticulación de la universidad con la agenda ambiental regional. Al respecto la acción de mejoramiento propuesta fue el desarrollo de una política ambiental institucional encaminada a disminuir los riesgos ambientales y a fomentar gestión ambiental entre la comunidad universitaria.

La política curricular de la Universidad de Caldas brinda espacios para desarrollar óptimamente procesos educativo-ambientales, siempre y cuando, éstos se entiendan desde su perspectiva holística, interdisciplinar y compleja, articulando aspectos biológicos, sociales, políticos, económicos, éticos y estéticos, mediante una interpretación crítica de la realidad y el diálogo constructivo de saberes y actores. Los enfoques del currículo propuestos comparten los ideales de lo educativo ambiental, específicamente en lo referente a que sea:

- Holístico: "entendido como la relación integral entre el todo y sus partes, en donde cada parte contiene potencialmente al todo y la totalidad refleja la unidad íntima de sus partes; de tal manera que se genere una concepción sistémica de los procesos investigativos en donde el estudio de un área específica del conocimiento influye y se relaciona de manera estructural con la totalidad de conocimiento; se propicie una potencial relación interdisciplinar y transdisciplinar de cualquier área del conocimiento y de la investigación; se superen los saberes cerrados e independientes para ser comprendidos como saberes abiertos e interdependientes de la unidad curricular global; y se propicie la existencia de un diálogo de saberes, por parte de profesores y estudiantes de las distintas disciplinas y áreas curriculares, para completar una visión global de cualquier problema cognitivo específico". 
- Cultural: "entendido como un proyecto intencionado para transformar el orden cultural existente. Una propuesta curricular puede entenderse como el proyecto cultural de una sociedad pues la institución educativa se creó con el propósito de asegurar los medios para garantizar las condiciones, las destrezas y los ideales que dan sentido y razón de ser a una sociedad".

- Interdisciplinario, "a partir de la convergencia de distintas disciplinas y perspectivas teóricas para el abordaje académico de todos los ejes problemáticos y temáticos que maneja la Universidad".

- Abierto, "referido a un conjunto armónico, articulado, ordenado, dinámico y productivo de acciones docentes, investigativas y proyectivas que permitan entender la universidad como un sistema que recibe, procesa, produce y transforma información en conocimientos científicos, artísticos y humanísticos".

- Orientado al desarrollo de competencias, "a partir de la promoción de aprendizajes teórico-conceptuales, éticos, valorales, sociales, praxiológicos y procedimentales atendiendo a la naturaleza de los objetos y campos del conocimiento y los contextos del propio aprendizaje y de la realidad en la cual se ha desenvolver cada sujeto que aprende".

La política curricular considera que las mediaciones pedagógicas utilizadas en los procesos formativos deben favorecer la capacidad crítica y reflexiva, la resolución de problemas esperados e inesperados y el desarrollo del pensamiento.

Con base en los documentos resultantes del proceso de resignificación curricular de la universidad se analizaron los objetos de estudio, la investigación y la proyección del programa bajo las categorías de interdisciplinariedad, enfoque científico y tecnológico, compromiso ético, perspectiva estética, enfoque social, enfoque ambiental explícito y compromiso con la transformación social; también se estudiaron los objetivos del programa, la identidad y las competencias formativas, bajo las categorías perspectiva o dimensión ambiental explícita, compromiso con lo social y la calidad de vida; transformación y el desarrollo social. Los resultados fueron los siguientes:

- Programas de la Facultad de Ciencias Agropecuarias: los objetos de estudio no evidencian compromiso ético y perspectiva estética, mientras que el enfoque científico y tecnológico es constante, el abordaje social y ambiental es apenas frecuente, siendo el compromiso con la calidad de vida, escaso. En los objetivos y la identidad formativa de los programas se aborda la temática ambiental y, en la mayoría, aspectos relacionados con la calidad de vida como el desarrollo social. En cuanto a las competencias formativas, el abordaje ambiental y el compromiso con la calidad de vida son frecuentes, mientras que el desarrollo social es escaso. Tanto en investigación como en proyección prima el enfoque científico-tecnológico.

- Programas de la Facultad de Ciencia Exactas y Naturales: la redacción del objeto de estudio contempla el énfasis científico-tecnológico de manera constante, al igual que los planteamientos de la investigación y la extensión de los programas; tanto en los objetivos, como en la identidad formativa la explicitación de los asuntos ambientales es frecuente.

- Programas de la Facultad de Ciencias para la Salud: tanto los objetos de estudio como las intencionalidades investigativas y de proyección, muestran un direccionamiento científico-tecnológico además de social; pese a que en los objetos de estudio los objetivos, las identidades formativas y las competencias formativas se presenta un compromiso con la calidad de vida, ello no se refleja en las intencionalidades de la investigación y la proyección. En todos los casos la alusión explícita a lo ambiental es escasa. 
- Programas de la Facultad de Artes y Humanidades: no se hace referencia específica a lo ambiental ni al quehacer interdisciplinar; tampoco se alude al compromiso con la calidad de vida ni con aspectos éticos; pese a ello, en los objetivos de los programas sí se contempla la calidad de vida.

- Programas de la Facultad de Ciencias Jurídicas y Sociales: en los objetos de estudio, la investigación y la proyección, se destaca el enfoque científico-tecnológico y el compromiso con lo social, lo que también es evidente en los objetivos, la identidad formativa y las competencias formativas en tanto muestran de manera frecuente alusiones a la calidad de vida y al desarrollo social.

- Programas de la Facultad de Ingeniería: el compromiso planteado es científico-tecnológico; en algunos programas se mencionan aspectos éticos, sociales y ambientales.

La combinación de las categorías mencionadas engloba la dimensión ambiental, por tal motivo podría considerarse que las facultades en las que con mayor frecuencia estaban presentes las categorías son justamente las que más se acercan a una verdadera inclusión de esta dimensión en su quehacer relativo a lo curricular. Las tres facultades con dicha característica son; la de Ciencias Agropecuarias, Ciencias para la Salud y Ciencias Jurídicas y Sociales. Las categorías que se encontraron con mayor frecuencia son: el enfoque científico-tecnológico y el aporte a la calidad de vida. En términos generales, las evidencias de interdisciplinariedad y las alusiones a los asuntos éticos, estéticos y ambientales son escasas.

Los programas de Biología, Enfermería, Licenciatura en biología y química; Licenciatura en educación básica con énfasis en educación física, recreación y deportes; Medicina y Tecnología en electrónica, incluyen en sus planes de estudio asignaturas específicas como aisladas para manejar los contenidos ambientales y educativoambientales, a saber; Ecología, Ecología y educación ambiental, Ecología humana y atención al ambiente.

El desarrollo explícito de los aspectos ambientales relacionados con el objeto de estudio de los diferentes programas de pregrado se realizan mediante asignaturas como; Planes de ordenamiento territorial, Fundamentos de ecología, Profundización en biodiversidad y recursos fitogenéticos, Derecho ambiental, Gestión ambiental, Gestión para la solución de problemas y conflictos ambientales, Geología ambiental, Saneamiento ambiental, Fauna silvestre, Ecología aplicada y ecodesarrollo, Química ambiental, Economía ecológica, Medio ambiente y desarrollo.

Los programas de pregrado en los cuales no se evidencia ninguna actividad académica relacionada, de manera genérica o profesionalizante, respecto al tema ambiental son: Antropología, Desarrollo familiar, Ingeniería en sistemas y computación, Licenciatura en artes escénicas con énfasis en teatro, Licenciatura en filosofía y letras, Licenciatura en lenguas modernas, Licenciatura en música, Sociología, Tecnología en administración judicial, Tecnología en finanzas, Tecnología en regencia de farmacia y Tecnología en sistemas informáticos.

La Universidad de Caldas ofreció desde 1997 una Licenciatura en educación ambiental, que en sus contenidos incluía enfoques ambientales desde lo cotidiano, lo social, lo institucional y lo natural. Los propósitos del programa eran: a) preparar educadores capaces de motivar a la comunidad por el respeto a todas las formas de vida y del medio ambiente donde habitan, como vía para optimizar la calidad de 
vida en el desarrollo del ser humano; b) formar educadores con criterios y actitudes de proyección futurista que planteen soluciones efectivas a la problemática ambiental desde el quehacer educativo, del proyecto educativo y desde su posición de gestores de la educación del futuro; c) desarrollar valores que le faciliten a las personas construir interiormente un equilibrio armónico en procura de una calidad integral de vida desde la ética civil; d) capacitar a las personas en el conocimiento necesario sobre el medio ambiente, para que identifiquen el deterioro de los sistemas naturales, y fomentar en ellos la habilidad para que consecuentemente diseñen, elaboren, ejecuten y evalúen programas de educación ambiental de acuerdo con las características del contexto social que aporten al desarrollo integral regional; y e) desarrollar la capacidad de análisis en la relación de interdependencia de los problemas ecológicos, la pedagogía y la educación ambiental. La Licenciatura dejó de ofrecerse porque estaba adscrita a la Facultad de Ciencias Agropecuarias y se consideró que ésta no correspondía con el objeto de estudio de la Facultad. Años después se reabre la licenciatura pero adscrita a la Facultad de Artes y Humanidades, con el objetivo de "formar profesionales con capacidad de diseñar y desarrollar programas en los niveles formal, no formal e informal en el área ambiental, fomentando el mejoramiento de la calidad de vida de los habitantes del país y la región de una manera sostenible". La primera cohorte inició estudios en el segundo período académico del año 2011.

En los programas de postgrado no se evidencia un compromiso con la inclusión de la dimensión ambiental; algunos de ellos abordan la temática por ser específica de su objeto de estudio, haciéndolo mediante asignaturas tales como; Economía ambiental, Gestión de recursos naturales, Sistema natural, Aprovechamiento de residuos agroindustriales, Economía ambiental, Economía aplicada a la gestión medioambiental, Evaluación económica de impactos ambientales, Evaluación macro de políticas ambientales, Legislación y normatización ambiental, Modelación de sistemas ecológicos, Orientaciones actuales del ordenamiento del territorio, Políticas del ordenamiento territorial, Economía agroecológica y Manejo de recursos naturales.

Respecto a los procesos investigativos, desde el enfoque ambiental se hace necesario resaltar algunos de los propósitos universitarios: a) proyectar como fortaleza la investigación para el estudio, el aprovechamiento, la conservación de la biodiversidad del medio ambiente social y natural, además del uso sostenible de los recursos naturales de la región y el país; b) fomentar el trabajo académico interdisciplinario, transdisciplinario e interinstitucional, apoyado en procesos permanentes de investigación; y c) aplicar los resultados de la investigación en las actividades de proyección, docencia, extensión y servicios de la Universidad.

La política de investigación ambiental colombiana reconoce como uno de sus nodos críticos la "falta de criterios y mecanismos claros de priorización que garanticen la pertinencia temática y regional, la relevancia y oportunidad de la investigación ambiental para la gestión ambiental" (Minambiente, 2001, p.19), asunto que también caracteriza los procesos investigativos de la Universidad de Caldas. Específicamente en relación con la investigación ambiental, al igual que como se diagnostica en la política nacional de este tipo de investigación, en la Universidad hay "poca valoración y reconocimiento institucional a la diversidad de modos de producción de conocimiento relevantes para el medio ambiente" (Minambiente, 2001, p.22) y, asimismo, poco reconocimiento de que se hace investigación ambiental.

La Proyección Universitaria de la Universidad de Caldas se encuentra reglamentada en su correspondiente política; en el preámbulo de la 
misma se hace alusión implícita a lo ambiental en tanto se le otorgan criterios de regulación ética a la proyección, así como un papel protagónico al diálogo de saberes con la sociedad civil y compromisos con el fortalecimiento de la esfera de lo público en pos de la contribución a solución de los problemas de la región y la nación.

La sostenibilidad y la gestión para la proyección, constituyen dos de los lineamientos de la política de proyección de la Universidad de Caldas; para la sostenibilidad se considera que la institución "a través de las acciones de proyección, contribuirá a desarrollar en la comunidad la capacidad de pensar, construir y mantener a lo largo del tiempo un espacio común de convivencia, teniendo en cuenta las dinámicas y las interdependencias que existen entre el mundo natural y el entorno construido, así como los diferentes valores políticos, económicos, sociales, culturales y ambientales que la comunidad forja históricamente y la identifican"; en cuanto a la gestión para la proyección se determina que "la universidad propiciará y facilitará la participación de su comunidad académica en diferentes escenarios culturales, tecnológicos, corporativos, empresariales, sociales y científicos, con el propósito de participar en la definición de políticas públicas de Estado, así como en la identificación de problemas a nivel local, regional y nacional, poner al servicio de éstos la capacidad humana, tecnológica, cultural y científica en la búsqueda de soluciones pertinentes a los contextos".

Desde la perspectiva universitaria, podría afirmarse que la ciudad de Manizales tiene un gran potencial para desarrollar procesos educativoambientales en educación formal y no formal, en proyección y en investigación; no obstante, también es necesario reconocer las deficiencias en el proceso formativo de los futuros profesionales, pues el abordaje de la multidimensionalidad del medio ambiente no es una característica destacable en los procesos formativos. En cuanto a la formación de maestros la situación es más preocupante aún, dado que en las diferentes licenciaturas brilla por su ausencia la formación ambiental, con lo cual, en un futuro cercano tendremos profesores que, aunque recién egresados, continuarán con las deficiencias en formación ambiental propias de los docentes actuales.

Si el objetivo es propiciar procesos educativo-ambientales transformadores y adecuadamente fundamentados, es necesario intervenir en el nivel educativo superior, pues sus egresados serán los responsables no sólo de las decisiones que se tomen en todos los ámbitos, sino también de formar niños y adolescentes comprometidos con la justicia social y el bienestar ecosistemático.

\section{A MANERA DE PROPUESTA}

Si bien podría pensarse en la intervención de las universidades en el nivel local, con miras a garantizar la inclusión de la multidimensionalidad de la temática ambiental en sus quehaceres investigativo, de proyección y de formación, parece más impactante proponer criterios para los procesos de acreditación de los programas, en tanto la inclusión de la dimensión ambiental en la formación de profesionales, podría jalonar el desarrollo de otros espacios comprometidos con el medio ambiente, pues "resulta indispensable fortalecer las comunidades académicas de disciplinas, profesiones, ocupaciones y oficios, como factor fundamental para alcanzar altos niveles de calidad en los distintos programas" (MEN, CNA, 2006, p.11). 
Al respecto el Consejo Nacional de Acreditación (CNA) propone la política de acreditación de los programas académicos y de las instituciones de educación superior, pues considera que la misma "es un medio importante para reconocer hasta dónde ese proceso se cumple satisfactoriamente y para establecer qué tanto la educación superior está respondiendo a las exigencias que le plantea el desarrollo del país". (MEN, CNA, 2006. p.11).

Para empezar, sería de gran valor que el CNA explicitara en la sustentación de los procesos de acreditación, la necesidad de vincular la educación superior con la permanente búsqueda de soluciones para la problemática ambiental, entendida desde sus múltiples perspectivas, lo cual tendría perfecta cabida en la idea de "formar profesionales, en distintas áreas y campos de acción, que enfrenten nuevos retos derivados de los avances científico-técnicos y de la explicitación de necesidades sociales previamente desatendidas o desconocidas".(MEN, CNA, 2006, p.11).

Hoy en día no se concibe un proceso formativo de calidad si en él no está incluida la dimensión ambiental, puesto que se entendió claramente que el medio ambiente es natural y social, lo cual lo convierte en un concepto que inevitablemente debe permear el quehacer formativo, investigativo y de proyección. Para el CNA (2006) la calidad del servicio público de educación superior:
"hace referencia a la síntesis de características que permiten reconocer un programa académico específico o una institución de determinado tipo y hacer un juicio sobre la distancia relativa entre el modo como en esa institución o en ese programa académico se presta dicho servicio y el óptimo que corresponde a su naturaleza" (p.26).

Para conceptuar sobre ese óptimo, el CNA definió un conjunto de características generales de calidad, centro de la propuesta que se presenta y que actúa como fundamento de los tres pasos de la acreditación de alta calidad, a saber: a) autoevaluación, b) evaluación externa o evaluación por pares y c) evaluación final. El proceso de acreditación de los programas tiene entre sus objetivos: a) propiciar el mejoramiento de la calidad de la Educación Superior, b) propiciar la idoneidad y la solidez de programas académicos de educación superior, c) señalar un paradigma de calidad a los programas de educación superior colombianos y d) promover en las instituciones la verificación del cumplimiento de su misión, sus propósitos y sus objetivos en el marco de la Constitución y la Ley, y de acuerdo con sus propios estatutos (MEN, CNA, 2006, p.38). Para el logro de estos objetivos es clave la existencia del compromiso institucional con el fin de llevar a la práctica los lineamientos propuestos, tanto en la Política de Investigación Ambiental, como en la de educación de este tipo, ganando coherencia con el hecho de que el CNA (2006)

Pone de relieve el ser la educación superior un espacio de búsqueda y transmisión del conocimiento que debe desenvolverse en un ambiente de convivencia, de paz y de libertad y dentro de un marco democrático, participativo y pluralista y destaca el papel de la educación superior en el logro de la unidad nacional, en afianzar entre los ciudadanos el respeto a la dignidad humana y la vigencia plena de los derechos humanos, en construir una actitud consciente para la preservación del medio ambiente y 
en ser factor esencial para el desarrollo integral de los colombianos (p.45).

Según el CNA el Sistema de Acreditación Nacional opera con base en diez criterios, a saber: universalidad, integridad, equidad, idoneidad, responsabilidad, coherencia, transparencia, pertinencia, eficacia y eficiencia. En este contexto, y como parte del criterio de responsabilidad ${ }^{4}$, bien podría incluirse la relacionada con el medio ambiente con el fin de darle soporte a una serie de características e indicadores capaces de promover la formación de profesionales comprometidos con el entendimiento de la complejidad del mismo y, por ende, con la complejidad e interdisciplinariedad propia de su estudio e intervención. Asimismo, la pertinencia daría cabida a este aspecto pues se define como "la capacidad de la institución y su programa para responder a necesidades del medio"(MEN, CNA, 2006, p.44); no obstante, sería ideal la explicitación de las necesidades ambientales sentidas o no, por parte de los diferentes estratos de la sociedad.

Los factores que el CNA ha definido para la realización de los procesos de acreditación de alta calidad de los programas académicos son: 1) misión y proyecto institucional, 2) estudiantes, 3) profesores, 4) procesos académicos, 5) bienestar institucional, 6) organización, administración y gestión, 7) egresados e impacto sobre el medio y 8) recursos físicos y financieros. Cada uno de los factores tiene unas características específicas y unos indicadores que permiten determinar su grado de cumplimiento.

A continuación se presenta una propuesta de ajuste para algunas características e indicadores que podrían dar cuenta de la necesaria inclusión de la dimensión ambiental en los procesos formativos de los futuros profesionales. Para evitar una gran extensión del escrito, sólo se presentan los aspectos nuevos que deberían considerarse ${ }^{5}$.

En la característica 1, perteneciente al factor 1, se considera como uno de los aspectos a evaluar la "correspondencia entre la misión y los principios y objetivos establecidos por la ley para la educación superior" (p.54). Teniendo en cuenta que en la ley de Educación Superior se hace explícito el objetivo de "promover la preservación de un medio ambiente sano y fomentar la educación y cultura ecológica" (Congreso de la República, 1992. Artículo 6), bien valdría la pena incluir un indicador referente al grado de correspondencia entre la misión institucional y los objetivos de la educación superior planteados en la ley correspondiente.

La característica 2, del mismo factor, titulada Proyecto Institucional se enuncia así:

"el proyecto institucional orienta el proceso educativo, la administración y la gestión de los programas y sirve como referencia fundamental en los procesos de toma de decisiones sobre la gestión del currículo, de la docencia, de la investigación, de la internacionalización, de la extensión o proyección social y del bienestar institucional" (p.55)

A lo cual podría agregársele: en el marco de las características, situaciones y problemáticas socio-ambientales del país, lo cual llevaría a la formulación de un indicador específico: existencia y aplicación de políticas institucionales para orientar las acciones y decisiones del programa académico en lo relacionado con el impacto ambiental de su quehacer. 
La característica 3, denominada proyecto educativo del programa, tiene como uno de sus indicadores el "grado de correspondencia entre el proyecto educativo del programa y el proyecto institucional" (p.58). Si en el proyecto institucional se buscara la explicitación del compromiso ambiental este indicador automáticamente lo estaría incluyendo. No obstante, el aspecto a evaluar "orientaciones en el proyecto educativo del programa para desarrollar las funciones de la docencia, la investigación, la extensión o proyección social y la internacionalización" (p.57) podría complementarse con las palabras: y el impacto ambiental del quehacer profesional.

La relevancia académica y pertinencia social del programa constituyen la característica 4; uno de los aspectos que propone para evaluación es "políticas académicas de la institución y orientaciones del programa en relación con las necesidades del entorno" (p.59), para lo cual se define como indicador "número y tipo de actividades del programa que muestran la relación del plan curricular con las necesidades locales, regionales, nacionales e internacionales" (p.59). Aunque es obvio que en las necesidades mencionadas están incluidas las ambientales, para ganar en claridad y evitar olvidos indeseados el indicador podría mejorarse aclarando los tipos de necesidades que deberían contemplarse, por ejemplo, sociales, políticas, ambientales, económicas, etc.

En la característica 8, del factor 2 (estudiantes), denominada participación en actividades de formación integral y explicada como;

"el programa promueve la participación de los estudiantes en actividades académicas, en proyectos de investigación, en grupos o centros de estudio, en actividades artísticas, deportivas y en otras de formación complementaria, en un ambiente académico propicio para la formación integral" (p.64)

Podría incluirse la evaluación del cumplimiento de la estrategia Proyecto Ambiental Universitario propuesto en la Política Nacional de Educación Ambiental.

A la característica 18, relacionada con la integralidad del currículo, perteneciente al factor 4 (procesos académicos) que se enuncia como;

"el currículo contribuye a la formación en valores, actitudes, aptitudes, conocimientos, métodos, principios de acción básicos y competencias comunicativas y profesionales, de acuerdo con el estado del arte de la disciplina, profesión, ocupación u oficio, y busca la formación integral del estudiante, en coherencia con la misión institucional y los objetivos del programa"(p.79)

Sólo faltaría agregarle en la última oración, "en coherencia con el derecho a un ambiente sano".

Algunos de los aspectos a evaluar en esta característica son "actividades que contempla el programa para la formación o desarrollo de habilidades para el análisis de las dimensiones ética, estética, económica, política y social de problemas ligados al programa" (p.79) y "formación científica, estética y filosófica" (p.80). En ambos casos la mención de la dimensión ambiental brilla por su ausencia. En el plano concreto de los indicadores, uno de los que se propone es: "porcentaje de actividades distintas a la docencia y la investigación dedicadas al 
desarrollo de habilidades para el análisis de las dimensiones ética, estética, filosófica, científica, económica, política y social de problemas ligados al programa, a las cuales tienen acceso los estudiantes" (p.79) el cual podría complementarse con el análisis de la dimensión ambiental. También podría lograrse con un nuevo indicador: número y tipo de actividades académicas desarrolladas dentro del programa, en las que se analizan las diferentes tendencias de la situación y la problemática socio-ambiental a nivel nacional e internacional.

En la característica 28 , extensión o proyección social, enunciada como "el programa ha definido mecanismos para enfrentar académicamente problemas del entorno, promueve el vínculo con los distintos sectores de la sociedad e incorpora en el plan de estudios el resultado de estas experiencias" (p.94) se tiene como uno de los indicadores "existencia de criterios y políticas institucionales y del programa en materia de extensión o proyección social" (p.95) que bien podría complementarse con criterios y políticas institucionales de corte ambiental.

Otra característica del factor procesos académicos es la relacionada con los recursos de apoyo docente, cuyo enunciado es:

\begin{abstract}
"el programa, de acuerdo con su naturaleza y con el número de estudiantes, cuenta con recursos de apoyo para el desarrollo curricular tales como talleres, laboratorios, equipos, medios audiovisuales, sitios de práctica, estaciones y granjas experimentales, los cuales son suficientes, actualizados y adecuados." (p.99)
\end{abstract}

Para lo cual contempla como algunos de los aspectos que se deben evaluar: a) laboratorios y talleres suficientemente dotados con equipos, además de materiales adecuados y actualizados, según la naturaleza, metodología y exigencias del programa, que cumplen las normas sanitarias y de bioseguridad previstas en la ley o en los reglamentos; b) acatamiento de normas sobre bioseguridad, tratamiento de desechos tóxicos y prevención de accidentes; c) acatamiento de la legislación nacional e internacional en materia de prácticas con animales. El problema de esta característica radica en que tales aspectos de evaluación no fueron llevados a indicadores, lo cual propicia que se queden en letra muerta. La idea sería la verificación del cumplimiento de las normas sanitarias, ambientales y de bioseguridad en todo tipo de prácticas de los profesionales en formación.

En la característica 33, organización, administración y gestión del programa, enunciada como: "la organización, administración y gestión del programa favorecen el desarrollo y la articulación de las funciones de docencia, investigación, extensión o proyección social y la cooperación internacional" (p.103) debería agregarse: "y la conservación del medio ambiente" lo cual modificaría en el mismo sentido los dos primeros indicadores.

En la característica 37, influencia del programa en el medio, enunciada como: "en el campo de acción del programa, éste ejerce una influencia positiva sobre su entorno, en desarrollo de políticas definidas y en correspondencia con su naturaleza y su situación específica; esta influencia es objeto de análisis sistemático" (p.108) muy bien quedaría un indicador que diera cuenta de información sobre el impacto ambiental de los proyectos que el programa haya desarrollado o contribuido a desarrollar. 
Aunque parecieran pequeñas y puntuales estas modificaciones, es altamente probable que su implementación, por lo menos, generara en los programas que se someten a acreditación de alta calidad un momento de reflexión y de análisis sobre su verdadera posición frente a la problemática ambiental. Es bien sabido que la perspectiva ambienta es crítica, y su entrada en la universidad apuntalaría la incondicionalidad de la Universidad que, en palabras de Derrida:

[...] al ser incondicional, semejante resistencia podría oponer la universidad a un gran número de poderes: a los poderes estatales (y, por consiguiente, a los poderes políticos del Estado-nación así como a su fantasma de soberanía indivisible: por lo que la universidad sería de antemano no sólo cosmopolítica, sino universal, extendiéndose de esa forma más allá de la ciudadanía mundial y del Estado-nación en general), a los poderes económicos (a las concentraciones de capitales nacionales e internacionales), a los poderes mediáticos, ideológicos, religiosos y culturales, etc., en suma, a todos los poderes que limitan la democracia por venir [...].(Derrida, 2002, p. 14)

\section{BIBLIOGRAFÍA}

- Colombia. Congreso de la República. Ley 30 de 1992. Recuperado de http://www.mineducacion.gov.co/1621/article-86437.html

- Colombia. Ministerio del Medio Ambiente y Ministerio de Educación Nacional. Política Nacional de Educación Ambiental. (2002). Recuperado de http://www.humboldt.org.co/.../politica/politicas_ambientales/

- Colombia. Ministerio del Medio Ambiente. Consejo Nacional Ambiental. Departamento Nacional de Planeación. Colciencias. (2001). Política Nacional de Investigación Ambiental. Recuperado de http://www.humboldt.org.co/.../politica/politicas_ambientales/

- Derrida, Jaques. (2002). La universidad sin condición. Madrid: Trotta.

- Ministerio de Educación Nacional. (1994). Memorias del seminario internacional "La dimensión ambiental y la escuela". Santafé de Bogotá: El Ministerio, FES,

- Universidad Autónoma de Manizales. Información institucional recuperada de http://www.autonoma.edu.co/

- Universidad Católica de Manizales. Información institucional recuperada de http://www.ucm.edu.co/

- Universidad de Caldas. Información institucional recuperada de http://www.ucaldas.edu.co/

- Universidad de Caldas. Plan de desarrollo 2009-2018. Manizales: la Universidad, (2009).

- Universidad de Manizales. Información institucional recuperada de http://www.manizales.unal.edu.co/

- Universidad Nacional de Colombia. Sede Manizales. Información institucional recuperada de http://www.umanizales.edu.col

1. Médica epidemióloga, Magister en Educación, Profesora Titular de la Universidad de Caldas

2. Los diferentes artículos derivados de esta investigación se encuentran en http://lunazul.ucaldas.edu.co 
3. La información puede confrontarse en: http://www.manizales.unal.edu.co/ http://www.autonoma.edu.col

http://www.ucm.edu.co/http://www.umanizales.edu.co/

http://www.ucaldas.edu.col

4. En el texto se define responsabilidad como: "la capacidad existente en la institución y su programa para reconocer y afrontar las consecuencias que se derivan de sus acciones. Tal capacidad se desprende de la conciencia previa que se tiene de los efectos posibles del curso de acciones que se decide emprender. Se trata de un criterio íntimamente relacionado con la autonomía aceptada como tarea y como reto y no simplemente disfrutada como un derecho" (COLOMBIA, MEN, CNA, 2006. p.44).

5. El documento completo que contiene los lineamientos para la acreditación de los programas puede consultarse en: http://www.cna.gov.co/1741/article-186377.html 